\section{G241(P) CIDOFOVIR IN SEVERE ADENOVIRUS INFECTION IN AN IMMUNOCOMPETENT CHILD: A CASE REPORT AND REVIEW OF THE LITERATURE}

${ }^{1} \mathrm{E}$ Brettle, 'J Alexander, ${ }^{1} \mathrm{C}$ Kanaris, ${ }^{2} \mathrm{M}$ Semple. 'Paediatric Intensive Care, University Hospital North Midlands Trust, Stoke-on-Trent, UK; ${ }^{2}$ Faculty of Health and Life science, University of Liverpool, Liverpool, UK

\subsection{6/archdischild-2020-rcpch.208}

Adenovirus infection is self-limiting in immunocompetent children but can be severe and potentially fatal in immunocompromised children. Cidofovir is used in severe Adenovirus infection in immune compromised paediatric patients as standard practice but its use in immunocompetent children with severe disease is not well described.

We used Cidofovir successfully in a 5-month-old infant with severe Adenovirus infection. Born at 30 weeks gestation weighing $475 \mathrm{~g}$, she suffered from chronic lung disease and presented with a clinical bronchiolitis illness proven to be due to adenovirus. The infection progressed rapidly to severe lung disease needing nitric oxide, high frequency ventilation and inotropes. Initial Adenovirus viral load was 43,000 copies and following MDT discussion and counselling of the family, a $5 \mathrm{mg} / \mathrm{kg}$ once weekly course of Cidofovir was administered. Her viral load increased to a maximum of 444,000 copies but this improved to $<400$ following treatment. No nephrotoxicity was experienced; she recovered from her illness and was discharged to her local hospital

We undertook a systematic review of the published evidence of the use of Cidofovir in children and identified 40 articles of which 29 were relevant. The majority of literature reports the use of Cidofovir in immune compromised children with Adenovirus or CMV infection. There was limited evidence of its use in immunocompetent children. The mortality rate of severe adenovirus infection is high in children who are immune compromised, but no clear evidence of benefit in Cidofovir use was identified in the paediatric studies reviewed. A recent report of early use in adenovirus infection in 7 immunocompetent adults suggested evidence of possible benefit.

A common side-effect of Cidofovir is nephrotoxicity, with up to $28 \%$ of patients reported to develop evidence of renal dysfunction. Alternative dosing regimens are recommended if patients are nephropathic or at high risk of acquired renal injury

We weighed the potential benefit of treatment against the risk of nephrotoxicity in our patient and successfully treated a potentially fatal illness. We believe that Cidofovir could be used in immunocompetent patients with severe adenovirus infection but acknowledge that further research evidence is required before this treatment can be recommended.

\section{G242(P) DOES THE SEPSIS SCREENING TOOL USED IN THE PAEDIATRIC EMERGENCY DEPARTMENT HIGHLIGHT CHILDREN AT RISK OF SEPSIS AND WHAT IS THE RELATIONSHIP OF INITIAL BPEWS SCORE TO PATIENT OUTCOME}

M Janks, F Cantle, E Ryan, H Joyce. Paediatric Emergency Department, Kings College Hospital, London, UK

10.1136/archdischild-2020-rcpch.209
Aims This study of children seen in the paediatric emergency department set out to determine how useful the sepsis screening tool was in alerting clinicians to unwell potentially septic children, and to establish if the initial BPEWS (beside paediatric early warning score) was predictive of admission.

Methods A retrospective study of the electronic patient records for patients who had triggered on the sepsis screening tool over a 4 month period. Information gathered included initial BPEWS with breakdown for each parameter, and the outcome - discharged or admission (admission included children observed in the clinical decision unit or use of the nursing outreach service).

Of those who required admission, further information was gathered as to final diagnosis, whether antibiotics were given (IV or oral) and the microbiology results.

Results In the study period 719 patients triggered on the sepsis screening tool of which 713 were analysed. 172 of these were admitted (24\%), and of these only 1 had an invasive bacteraemia.

Respiratory infections were a common cause of illness with $19 \%$ (33) having upper respiratory tract infection and $17 \%$ (29) having lower. 16\% (27) of those admitted were ultimately diagnosed with viral induced wheeze. 63 (37\%) were positive for one or more viruses on respiratory or stool samples.

Correlation analysis of the BPEWS showed that the higher the initial BPEWS, the more likely that children would be admitted. There was a correlation of $95 \%$ between these variables and chi squared analysis of the relationship showed a significant $\mathrm{p}$ value of $<0.01$.

Conclusion Whilst large numbers of children triggered on the sepsis screening tool, $76 \%$ were not admitted and thus raises questions as to the sensitivity of the tool. Increasing BPEWS was found to be predictive in identifying children who require admission. This suggests that perhaps the tool requires some adaptations, such as using high BPEWS as a significant trigger, to allow it to be more sensitive and applicable for this population and workforce.

\section{G243(P) WOULD THE INTRODUCTION OF KETAMINE SEDATION FOR FACIAL WOUND CLOSURE IN THE PAEDIATRIC EMERGENCY DEPARTMENT REPRESENT PRUDENT HEALTHCARE?}

${ }^{1} \mathrm{~L}$ Potter, ${ }^{2} \mathrm{~J}$ Morgan. 'School of Medicine, Cardiff University, Cardiff, UK; ${ }^{2}$ Paediatric Emergency Department, University Hospital Wales, Cardiff, UK

\subsection{6/archdischild-2020-rcpch.210}

Aims Facial lacerations are a common presentation to the Paediatric Emergency Department (PED). Wound closure with sutures can be challenging in children due to agitation, therefore non-compliant children may need to be sutured under general anaesthesia (GA). Ketamine is a safe alternative to GA and would drastically reduce hospital admissions and associated costs for this procedure. We aimed to establish whether the introduction of a Ketamine sedation list would represent prudent healthcare; both economically and in terms of optimising resources without compromising patient safety.

Methods Children 0-15 years referred to Maxillofacial surgeons (OMFS) with facial lacerations for wound closure over a 12-month period $(01 / 04 / 18$ - 01/04/19) were identified using hospital coding. Information of their injury and 